\title{
Characterisation of the Polyphenol Content in the Kiwifruit (Actinidia deliciosa) Exocarp for the Calibration of a Fruit-sorting Optical Sensor
}

\author{
Patrizia Pinelli, ${ }^{a}$ Annalisa Romani, ${ }^{a}$ Elisa Fierini, ${ }^{a}$ Damiano Remorini ${ }^{b}$ \\ and Giovanni Agatic
}

\begin{abstract}
:
Introduction - Kiwifruit contains high amounts of anti-oxidants beneficial to health. Its quality is influenced by ripening time, genotype, cultivation techniques, climate and storage conditions after harvest.

Objective - The aim of the present study was to characterise the phenolic content by HPLC methods and to evaluate the performance of a portable optical sensor (Multiplex 3), for in vivo non-destructive phenolic compound assessment in kiwifruits.

Methods - Kiwifruits peel extracts were characterised by reverse-phase (RP) HPLC with diode-array detector (DAD) and electrospray ionisation (ESI) with MS using the Zorbax SB-Aq. column from Agilent. The fluorimetric sensor method is based on the screening of fruit chlorophyll fluorescence excitation and allows the UV absorbance of intact fruit skin to be measured. The flavonol index, $F L A V$, was calculated as $\log \left(F R F_{\mathrm{R}} / F R F_{\mathrm{UV}}\right)$, where $F R F_{\mathrm{R}}$ and $F R F_{\mathrm{UV}}$ are the chlorophyll fluorescence excited with red and UV light.

Results - Hydroxycinnamic acids, procyanidins, and quercetin glycosides were the main polyphenol classes detected by HPLCDAD-ESI/MS in the kiwifruit skin. A good linear regression $\left(R^{2}=0.88\right)$ was found between the fluorimetric sensor FLAV index and flavonol chromatographic analysis of the fruits. The FLAV index was able to detect the higher content of flavonols in sun-exposed fruits with respect to mid-shaded and shaded ones in accordance with the destructive analysis.

Conclusion - The fluorimetric sensor represents a rapid and non-invasive tool to: (i) monitor the flavonol accumulation in kiwifruit and to assess its quality concerning the healthy anti-oxidant properties; (ii) evaluate the effect of environmental and agronomical factors related to the fruit quality; and (iii) select fruits with the largest flavonol content, and consequently less susceptible to pathogen attack, in order to improve their storage durability. Copyright $\odot 2013$ John Wiley \& Sons, Ltd.
\end{abstract}

Keywords: HPLC-DAD-ESI/MS; flavonols; fluorescence; fruit quality; Actinidia deliciosa

\section{Introduction}

The kiwifruit is the edible berry of several Actinidia species. The well known commercially available variety, the green-fleshed kiwifruit, is the 'Hayward' cultivar, which belongs to the Actinidia deliciosa species. Kiwifruit contains high amounts of healthbeneficial anti-oxidants, such as vitamin C, vitamin $\mathrm{E}$, carotenoids and polyphenolic compounds. The polyphenols include chlorogenic and other caffeic acid derivatives, coumaric acid, protocatechuic acid, quercetin glycosides, catechin, epicatechin and procyanidins, naturally occurring in free form, bound to protein or polysaccharides, or in a polymeric form (SunWaterhouse et al., 2009).

A specific analysis of different vegetal tissues (peel and pulp) has shown that peel crude extract contains tocopherols, sterols, triterpene, chlorogenic acid and flavonoids, whereas pulp raw extracts led to the isolation of caffeic acid glucosyl derivatives and coumarin glycosides in addition to the above compounds compounds (Fiorentino et al., 2009). A recent study reported that kiwifruit, more rich in polyphenols compared with other fruits such as orange, grapefruit and apple, has important antioxidant activities in vitro: in particular, inhibitory effects on lipid oxidation, a $\mathrm{H}_{2} \mathrm{O}_{2}$-eliminating property and a superoxide dismutase (SOD)-like activity (Iwasawa et al., 2011). The antioxidant potential of Actinidia fruits was found to be largely influenced by vitamin C and total polyphenol levels, but also dependent on species and cultivars (Du et al., 2009). The kiwifruit quality depends on several factors such as ripening time, genotype, cultivation techniques and environmental conditions (Kempler et al., 1992; Snelgar et al., 1998; Ferguson, 1999). The polyphenol content of kiwifruits was also influenced by light exposure, harvest time and storage conditions during the postharvest (Montanaro et al., 2007; Tavarini et al., 2008).

Because of this, the availability of a rapid screening tool for polyphenol content of kiwifruits, and of cultivated fruits in general, could represent an innovative useful technique in phytochemical analyses. Optical methods for the evaluation of fruit quality are of great interest because they are

\footnotetext{
* Correspondence to: P. Pinelli, Department of Pharmaceutical Sciences, University of Florence, Via Ugo Schiff 6, 50019, Sesto Fiorentino, Firenze, Italy. Email: patrizia.pinelli@unifi.it

a Department of Pharmaceutical Sciences, University of Florence, Via Ugo Schiff 6, 50019, Sesto Fiorentino, Firenze, Italy

b Dipartimento di Scienze Agrarie, Alimentari e Agro-ambientali, University of Pisa, Via del Borghetto 80, 56124, Pisa, Italy

c Istituto di Fisica Applicata 'Nello Carrara' IFAC, Consiglio Nazionale delle Ricerche, Via Madonna del Piano 10, 50019, Sesto Fiorentino, Firenze, Italy
} 
non-destructive, repeatable over time on the same targets and rapid, and therefore are applicable to a large number of samples. Internal properties of kiwifruits can be assessed nondestructively by reflectance and interactance of visible-nearinfrared radiation (McGlone et al., 2002).

Reflectance-spectroscopy-based methods have been used to determine pigments on a fruit surface (Merzlyak et al., 2003), but failed in the detection of colourless flavonols (Hagen et al., 2006). In contrast, epidermal flavonols were detected in situ by applying the chlorophyll (Chl) fluorescence screening method (Bilger et al., 1997; Agati et al., 2007) in which UV-absorbing phenolic compounds present in leaf epidermises and fruit skins filter part of the excitation light during its path towards the underlying Chl. This technique was recently employed to define a non-destructive flavonol index in apple peels by using a portable fluorescence sensor (Betemps et al., 2012). The fluorescence screening method is particularly suitable to investigate in situ absorbance of skin in fruits that retain chlorophyll in the flesh, such as kiwifruits. Indeed, it was previously shown that chlorophyll fluorescence can be measured through the exocarp of whole kiwifruits (Remorini et al., 2011).

The use of non-destructive sensors for flavonols on kiwifruit can have different purposes: (i) as a sorting method of topquality fruits with high health benefit properties to be consumed fresh; (ii) to evaluate the effect of environmental and agronomical factors related to the fruit quality; and (iii) to control fruit storability.

A critical point in defining a non-destructive index to predict the concentration of a specific compound concerns its calibration against wet chemistry. For this, in the present study, we characterised at first the phenolic content of kiwifruit extracts by HPLC with diode-array detector and electrospray ionisation with MS (HPLC-DAD-ESI/MS) methods. Then, we used the results of destructive analysis to calibrate the fluorescencebased Multiplex (Force-A, Orsay, France) sensor to be used as a quality-sorting tool in kiwifruits.

\section{Experimental}

\section{Plant material}

Kiwifruits (A. deliciosa (A. Chev.) Liang \& Ferguson, cv 'Hayward') were collected from a commercial orchard in Rigoli (Pisa, Italy; $43^{\circ} 46^{\prime} 56.761^{\prime \prime} \mathrm{N}, 10^{\circ} 25^{\prime} 16.515^{\prime \prime} \mathrm{E}$ ) at the end of the 2010 growing season. Soluble solid content of kiwifruits ranged between 7.8 and $9.8^{\circ} \mathrm{Bx}$. Vines were trained as a free palmette at an altitude of $11 \mathrm{~m}$ above sea level. Fruits were harvested from positions in the plants exposed to different light conditions (full sun, mid-shade and shade). The full sun midday photosynthetic photon flux density measured at harvest was $1100 \mu \mathrm{mol} / \mathrm{m} 2 / \mathrm{s}$. Mid-shade and shade light regimes were $15 \%$ and $2 \%$ of full sun, respectively. Samples ( 25 fruits per light condition) were measured the same day of harvest by the fluorimetric sensor, stored at $0^{\circ} \mathrm{C}$ (Tavarini et al., 2008) for 3 months and, then, acclimated to ambient temperature before being measured again. During fruit collection, the most exposed side of each fruit was marked. Under shade condition, the most exposed side was defined as that facing the external part of the canopy.

\section{Multiplex fluorimetric sensor}

The Multiplex (Mx) fluorimetric sensor (Force-A, Orsay, France) is described in detail elsewhere (Ben Ghozlen et al., 2010). It measures fluorescence emitted by chlorophyll, in the red (RF) and far-red (FRF) spectral regions, under excitation with different light-emitting diode
(LED) sources in the UV ( $375 \mathrm{~nm}$ ) and visible (blue at $450 \mathrm{~nm}$, green at $515 \mathrm{~nm}$ and red at $630 \mathrm{~nm})$.

The intensity of the chlorophyll fluorescence (ChIF) emitted by a kiwifruit depends on the amount of excitation light able to reach the $\mathrm{Chl}$ pigment, that is on the transmittance of the peel ( $T_{\text {exocarp }}$ ) at the excitation wavelength (Mendes Novo et al., 2012). Flavonols in the exocarp can attenuate part of the incident radiation in the UV-A region before this can reach the $\mathrm{Chl}$ molecules. Consequently, the higher the peel's concentration of flavonols the lower the intensity of ChIF under UV excitation. Since long wavelength visible light is not absorbed by flavonols, ChlF excited by green-red wavelengths can be considered as a reference signal. Assuming $T_{\text {exocarp }}=1$ for red light, we can compare two ChlF signals under UV and red excitation to obtain an index proportional to the kiwifruit peel flavonoid content:

$$
\text { Flav }=\log \left(F R F_{R} / F R F_{\mathrm{uv}}\right)
$$

where $F R F_{\mathrm{R}}$ and FRF $\mathrm{UV}$ are the far-red ChIF excited in the red and UV, respectively. In this way, the FLAV index gives a non-destructive measure of the exocarp absorbance in the UV-A at $375 \mathrm{~nm}$ (Betemps et al., 2012).

All the kiwifruits were measured by the $M x$ sensor on both their flat sides through a $2 \times 3 \mathrm{~cm}$ mask with the longer length parallel to the longitudinal axis of the fruit.

\section{Extraction of polyphenols}

In order to cover the largest range of flavonols concentrations, the kiwifruits to be used to build the Mx calibration curve were selected on the basis of the FLAV values. Twenty-one samples with FLAV values between 0.5 to 1.1 (Mx units) were chosen and their exocarp analysed destructively. The exocarp of each kiwifruit side (exposed or not to sun) was peeled off and analysed separately. In detail, $2 \mathrm{~g}$ of skin wre ground under liquid nitrogen into fine powder. The resulting skin powder was transferred to $50 \mathrm{~mL}$ of acidified extraction solvent (ethanol:water $7: 3, \mathrm{v} / \mathrm{v}$, pH 3.2 adjusted by formic acid), maintained under stirring for $3 \mathrm{~h}$ and then filtered under vacuum. The pellet was re-extracted once again using the same procedure, and the final two pooled extracts were defatted twice with $50 \mathrm{~mL}$ of hexane and concentrated precisely to $2 \mathrm{~mL}$. The whole procedure was performed at room temperature $\left(20-25^{\circ} \mathrm{C}\right)$. Extracts were stored at $-20^{\circ} \mathrm{C}$ and, finally, centrifuged for $3 \mathrm{~min}$ at $14000 \times g$ before HPLC analysis.

\section{HPLC-DAD-ESI/MS analysis}

Analysis was carried out using a HP-1100 liquid chromatograph equipped with a DAD and a HP 1100 MSD API-electrospray (Agilent Technologies, Palo Alto, CA, USA). The mass spectrometer operated in negative ionisation mode (fragmentor $120 \mathrm{eV}$ ) to detect phenolic substances.

The column was a Zorbax SB-Aq. $4.6 \mathrm{~mm} \mathrm{ID} \times 150 \mathrm{~mm}(5 \mu \mathrm{m})$ from Agilent. The mobile phase was (A) water pH 3.2 acidified by formic acid and (B) acetonitrile. The following multi-step linear gradient was applied: from $100 \%$ to $85 \%$ of $A$ in $12 \mathrm{~min}$, which was maintained for $5 \mathrm{~min}$ $10 \mathrm{~min}$ to reach $75 \% \mathrm{~A}$, which was maintained for $8 \mathrm{~min}$, then $3 \mathrm{~min}$ to arrive at $0 \% \mathrm{~A}$, which was maintained for $2 \mathrm{~min}$ and $3 \mathrm{~min}$ to arrive at $100 \%$ A. Total time of analysis was $43 \mathrm{~min}$, flow rate $0.4 \mathrm{~mL} / \mathrm{min}$ and oven temperature $26 \pm 0.5^{\circ} \mathrm{C}$. UV-Vis spectra were recorded in the $190-600 \mathrm{~nm}$ range and the chromatograms were acquired at 280, 315, 330, 350 and $375 \mathrm{~nm}$.

\section{Polyphenols quantification}

Identification of individual phenols was carried out using their retention times, and both UV-Vis and MS spectra. The quantification of the single phenolic compounds was directly performed by HPLC-DAD using a five-point regression curve built with the available standards. Curves with a coefficient of determination $R^{2}>0.998$ were considered. In particular, caffeoyl acid amounts were calculated at $330 \mathrm{~nm}$ using chlorogenic acid as reference. Quercetin glycosides were calibrated at $350 \mathrm{~nm}$ using 
quercetin 3-O- $\beta$-D-glucoside as reference, and, finally, catechin oligomers were calculated at $280 \mathrm{~nm}$ using epicatechin as standard compound.

\section{Chemical reagents and materials}

Acetonitrile and hexane were provided by E. Merck (Darmstadt, Germany), water was purified with a Milli-Q system. Authentic standards of quercetin 3-O- $\beta$-D-glucoside (> 90\% HPLC), was purchased from Fluka (Germany Darmstadt), and of chlorogenic acid and epicatechin from Extrasynthese (Nord Genay, Lyon, France).

\section{Statistical analysis}

Statistical analysis was carried out with SigmaPlot Program 11.0 (Systat Software, Inc. Sigmaplot for Windows) and statistical differences between treatments were evaluated by a $t$-test analysis. Results are given as means \pm standard deviation (SD).

\section{Results and discussion}

\section{HPLC-DAD-ESI/MS analyses}

The main objective of the present work was to collect data on the skin polyphenol composition of kiwifruit by HPLC-DADESI/MS analyses and, then, to compare these results with those obtained by the optical method. To accomplish a complete characterisation of the kiwifruit, each compound belonging to different phenolic classes was quantified with the specific reference standard.

In the peel of kiwifruit, different classes of phenolic compounds were characterised: phenolic acid derivatives (as $\beta$-vanillic acid glucoside), coumarins, hydroxycinnamic acid derivatives; flavonols (quercetin 3-O- $\beta$-D-rutinoside and quercetin 3-O- $\beta$-D-rhamnoside), and procyanidins (mainly trimer and tetramer) with the corresponding monomers: catechin and epicatechin. The single compounds, as reported in Table 1, were identified using both retention times and their spectroscopic and spectrometric data, and referring to the literature when available.

For instance, the peak with $t_{\mathrm{R}}$ of $17.0 \mathrm{~min}$ showed a quasimolecular ion $[\mathrm{M}-\mathrm{H}]^{-}$at $\mathrm{m} / \mathrm{z} \quad 369$ and the following fragmentation pattern: $\mathrm{m} / \mathrm{z} 207$ corresponding to the loss of glucose $[\mathrm{M}-\mathrm{H}-162]^{-}$and $m / z 191$ consistent with the loss of a methylglucoside moiety $[\mathrm{M}-\mathrm{H}-162-15]^{-}$. This caffeic acid derivative was identified as the dimethyl caffeic acid hexoside, as previously reported in kiwifruit extracts (Sun-Waterhouse et al., 2009). It represented the main component of the extract for detection at $330 \mathrm{~nm}$. Moreover, the fragmentation pattern of the peaks at $t_{\mathrm{R}}$ of $13.7,16.7$ and 18.9 min was not completely recognised and, then, they were only tentatively identified as caffeic and ferulic acid derivatives. The detected flavonols, glycosides of quercetin, are also reported in Table 1.

Procyanidins and their related monomers, catechin and epicatechin, were another phenol class characterised in kiwifruit peel extracts. Procyanidins represent a class of polyphenol compounds found in several plant species, consisting of oligomers of flavan-3-ol units, most frequently linked either as $\mathrm{C4}-\mathrm{C} 6$ or $\mathrm{C4}-\mathrm{C} 8$. The presence of epicatechin, catechin and procyanidin (dimer and oligomer) has been documented previously on kiwifruit juice (Dawes and Keene, 1999). Procyanidin olygomers in kiwifruit extracts had a UV-Vis absorbance maximum at $280 \mathrm{~nm}$ and showed ESI/MS fragmentations at $\mathrm{m} / \mathrm{z} 1153,865$ depending on the grade of polymerisation (tetramers or trimers); the signal at $\mathrm{m} / \mathrm{z} 577$ corresponds to the loss of catechin units from both trimers and tetramers; the signal at $m / z 289$, related to catechin and epicatechin monomers, was also present (see Table 1). The presence of catechin and epicatechin was confirmed by comparison with commercial standards.

\begin{tabular}{|c|c|c|c|c|}
\hline & Compounds & $t_{\mathrm{R}}$ & MW & Negative ions $^{\mathrm{a}}(\mathrm{m} / \mathrm{z})$ \\
\hline \multirow[t]{2}{*}{ Phenols } & 6-hydroxy-7-( $\beta$-D-glucopiranosyloxy) coumarin & 11.6 & 340 & 339,177 \\
\hline & $\beta$-vanillic acid glucoside & 12.5 & 330 & 329,167 \\
\hline \multirow[t]{6}{*}{ Hydroxy-cinnamic acids } & Caffeic acid derivative & 13.7 & 356 & $355,297,191,179$ \\
\hline & Ferulic acid glucoside & 14.8 & 356 & 355,193 \\
\hline & Caffeic acid glucoside & 15.5 & 342 & 341,179 \\
\hline & Ferulic acid derivative & 16.7 & 370 & $369,193,179,135$ \\
\hline & Dimethyl caffeic acid hexoside & 17.0 & 370 & $369,207,191$ \\
\hline & Caffeic acid derivative & 18.9 & & $617,319,297,135$ \\
\hline \multirow[t]{2}{*}{ Flavonols } & Rutin & 25.7 & 610 & 609,301 \\
\hline & Quercetin 3-O- $\beta$-D-rhamnoside & 29.5 & 448 & 447,301 \\
\hline \multirow[t]{9}{*}{ Procyanidin } & Procyanidin trimer & 18.0 & 866 & 865,577 \\
\hline & Procyanidin tetramer & 18.4 & 1154 & $1153,865,577,289$ \\
\hline & Catechin & 18.8 & 290 & 289 \\
\hline & Epicatechin & 20.3 & 290 & 289 \\
\hline & Procyanidin trimer & 22.8 & 866 & 865,577 \\
\hline & Procyanidin tetramer & 24.2 & 1154 & 1153,577 \\
\hline & Procyanidin tetramer & 24.9 & 1154 & \\
\hline & Procyanidin trimer & 25.9 & 866 & \\
\hline & Procyanidin trimer & 26.7 & 866 & $865,527,491$ \\
\hline
\end{tabular}


The extraction procedure allowed the complete recovery of polyphenols. Even if the alkaline hydrolysis can liberate phenolic acids and improve the resolution of the polyphenol chromatographic profile (Sun-Waterhouse et al., 2009), the extraction procedure of the present work has been performed without the use of alkali, to avoid the degradation of flavan-3-ol oligomers (procyanidins), flavonols and hydroxycinnamic acid glycosides and derivatives.

Table 2 shows the HPLC-DAD quantitative results of the different polyphenol classes: flavonols, hydroxycinnamic acid derivatives and procyanidins, calibrated with the specific reference compounds, and acquired at the maximal absorbance wavelength, $350 \mathrm{~nm}, 330 \mathrm{~nm}$ and $280 \mathrm{~nm}$, respectively. Data are the mean $\pm S D$ of the concentrations obtained for each light condition (shade, mid-shade and full sun) and are reported as $\mathrm{mg} / \mathrm{g}$ of fresh weight.

Full sun exposure significantly increased the concentration of each polyphenol class with respect to mid-shade and shade light regimes. In particular, considering the mean values, the flavonols in fruits under full sun were $63.5 \%$ and $38.5 \%$ higher than the shade and mid-shade samples, respectively, as expected on the basis of their protective role in plants (Agati and Tattini, 2010). A light-induced increase in phenolic compounds of kiwifruit stalk was previously observed, but unfortunately the authors did not analyse the phenolic content in the fruit exocarps (Montanaro et al., 2007). It is well known that the activity of the key enzymes in the polyphenol biosynthetic pathway is stimulated by light exposure. Phenylalanine ammonia-lyase activity, simple phenol and flavonoids all increased when bagged apples were exposed to light (Ju et al., 1995). However, in other studies, only flavonoids (including anthocyanins) and not catechins or chlorogenic acid accumulated in larger amount in the most sunexposed fruits (Awad et al., 2001). Moreover, when grape berries cultivated in a shaded greenhouse were exposed to full sunlight (or sunlight with UV radiation removed), flavonols were increased while hydroxycinnamates decreased (Kolb et al., 2003). The same authors previously reported an opposite response of hydroxycinnamic acids to light in grape leaves (Kolb et al., 2001).

In our study, a 50\% higher concentration of hydroxycinnamic acid derivatives in sun-exposed fruits with respect to the shade ones was observed. Even for procyanidins, an increase of $38.7 \%$ in full sun samples was observed with respect to shade conditions, therefore, in the present study, light seems stimulate the synthesis of all polyphenol classes investigated.

The effect of light on the concentration of hydroxycinnamic acids on leaves and different fruits is controversial, it may depend on the particular skin structures (trichomes in kiwifruit) or the experimental light conditions (natural or greenhouse controlled) and needs further investigation to be elucidated.

\section{Calibration of the multiplex sensor}

In order to calibrate the Mx sensor, the HPLC analyses with detection at $375 \mathrm{~nm}$, one of the excitation wavebands of the optical sensor, were performed. The resulting chromatographic profile of kiwifruit peel extracts is shown in Fig. 1. It can be noted that, in addition to flavonols, a significant contribution to the $375 \mathrm{~nm}$ absorbance is given by the dimethyl caffeic acid hexoside $\left(t_{R}=17.0 \mathrm{~min}\right)$ and the caffeic acid derivative at $t_{R}=13.7 \mathrm{~min}$. Therefore, it was investigated to what degree variations in the UV-screening by the kiwifruit exocarp can be explained by changes in the skin phenolic content by plotting fluorometrically detected UV-A absorbance against absorbance of phenolics at $375 \mathrm{~nm}$, determined by HPLC analysis.

In Fig. 2A and 2B the relationship between the FLAV index and the total peak areas (normalised to the skin fresh weight) of flavonols and hydroxycinnamic acid derivatives, respectively, is represented. The association between FLAV and flavonol showed a good linear regression $\left(R^{2}=0.83\right)$. In contrast, the hydroxycinnamic acid derivatives contribution to the change of UV-A screening appears to be small $\left(R^{2}=0.35\right)$. Therefore, only flavonols and not hydroxycinnamic acid derivatives seem to exhibit a significant positive correlation with skin UV-A absorbance.

In Fig. 3 the FLAV index is plotted against the flavonol concentrations quantified by HPLC with detection at $350 \mathrm{~nm}$, close to

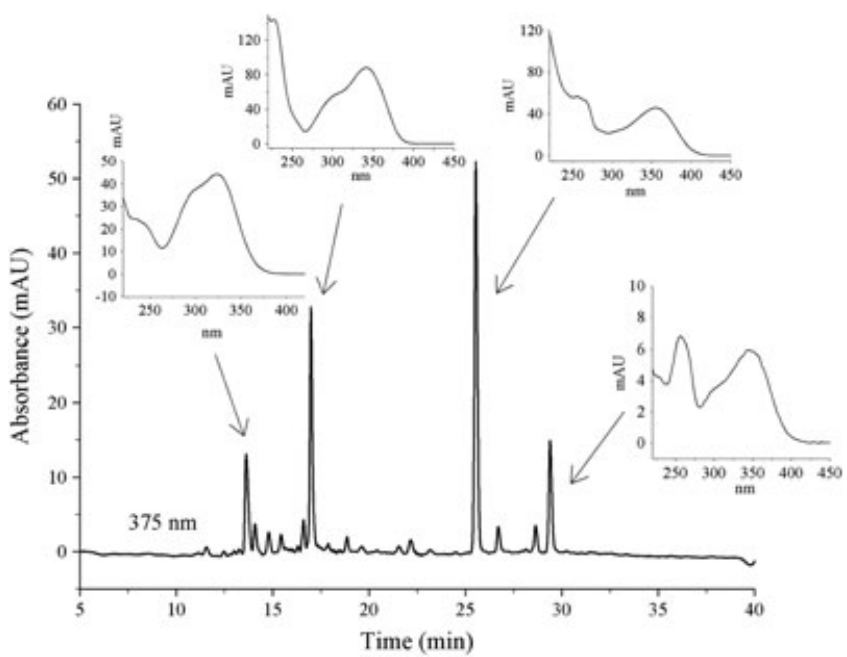

Figure 1. HPLC-DAD chromatographic profile (recorded at $375 \mathrm{~nm}$ ) of a kiwifruit peel hydroalcoholic extract. The UV-Vis spectra of the main phenol compounds: caffeic acid derivative $\left(t_{R}=13.7 \mathrm{~min}\right)$, dimethyl caffeic acid hexoside $\left(t_{R}=17.0 \mathrm{~min}\right)$, rutin $\left.t_{R}=25.7 \mathrm{~min}\right)$ and quercetin 3-O- $\beta$-D-rhamnoside $\left(t_{R}=29.5 \mathrm{~min}\right)$ are also reported.

Table 2. HPLC-DAD quantitative analysis of polyphenol classes identified in raw hydroalcoholic extracts of kiwifruit skin. Data are the average $( \pm S D)$ of the determinations acquired for each light conditions $(n=8-11)$ and are expressed as mg/g of fresh weight ${ }^{a}$

\begin{tabular}{lccc} 
Light regime & Flavonols & Hydroxycinnamic acids & Procyanidins \\
Shade & $0.054 \pm 0.018^{\mathrm{b}}$ & $0.374 \pm 0.159^{\mathrm{b}}$ & $5.804 \pm 2.647^{\mathrm{b}}$ \\
Mid-shade & $0.091 \pm 0.043^{\mathrm{b}}$ & $0.404 \pm 0.118^{\mathrm{b}}$ & $5.805 \pm 1.958^{\mathrm{b}}$ \\
Full sun & $0.148 \pm 0.060^{\mathrm{a}}$ & $0.747 \pm 0.241^{\mathrm{a}}$ & $9.470 \pm 2.105^{\mathrm{a}}$ \\
a For each class of compounds, values with different letters are significantly different at $p<0.05$ & \\
\hline
\end{tabular}



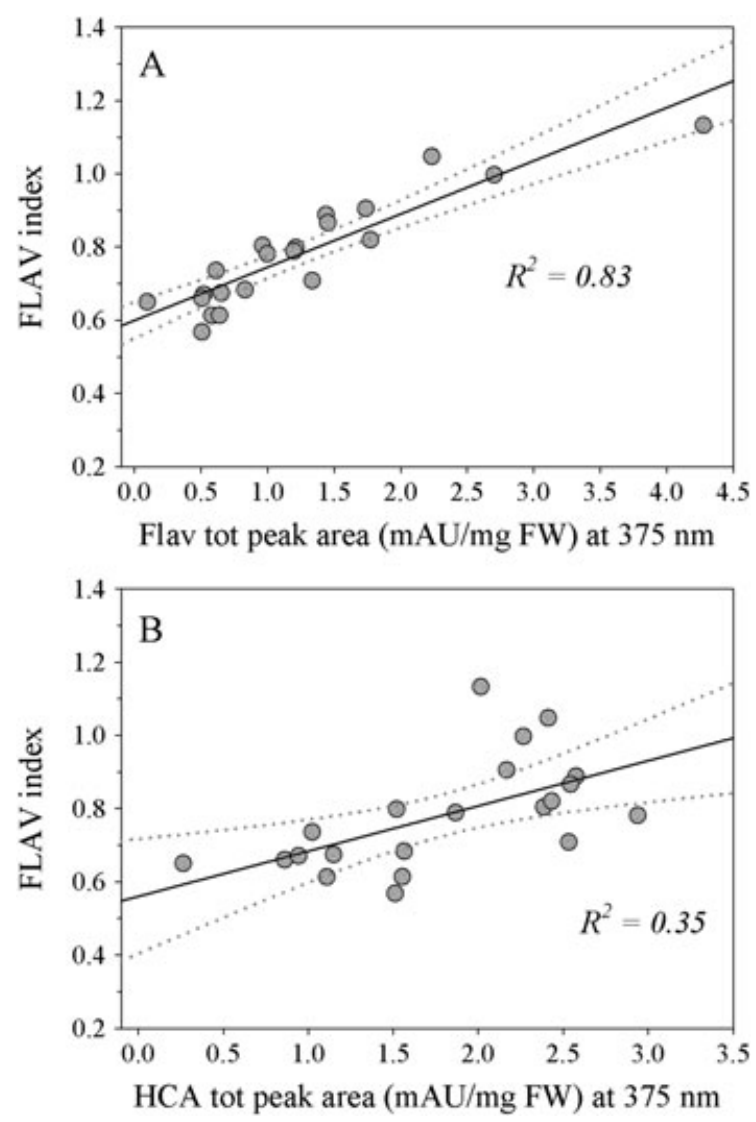

Figure 2. Relationship between the Multiplex FLAV index and the (A) flavonol (Flav) and (B) hydroxycinnamoyl derivatives (HCA) total peak areas obtained in the HPLC analysis of kiwifruit exocarp extracts recorded at $375 \mathrm{~nm}$, the same wavelength used for Mx detection. The solid lines indicate the linear fitting curves and the dotted lines indicate the $95 \%$ confidence limits.

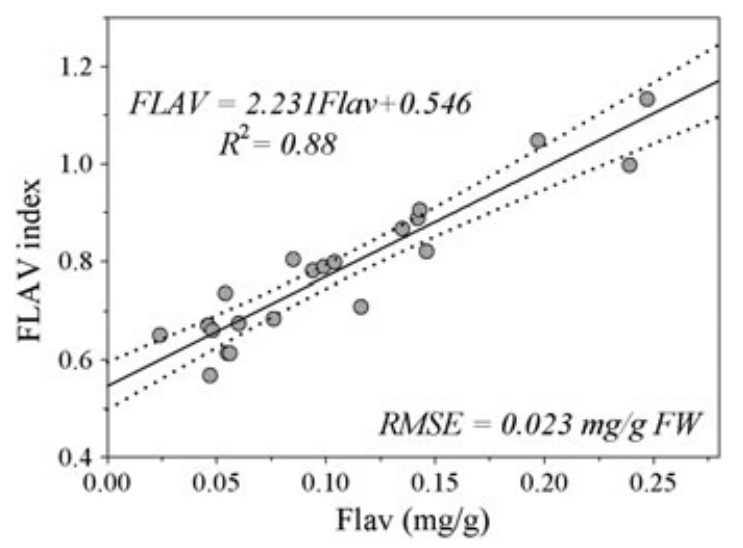

Figure 3. Calibration curve for the Mx FLAV index obtained through the actual flavonols (Flav) kiwifruit exocarp content quantified by HPLC using detection at $350 \mathrm{~nm}$. The solid line indicates the linear fitting curve and the dotted lines indicate the $95 \%$ confidence limits.

the maximum of the flavonol absorption spectrum. The regression line obtained was $\mathrm{FLAV}=0.55+2.23$. Flav, with $R^{2}=0.88$. The inverted equation applied to the same dataset produced the estimated flavonol values with root mean square error
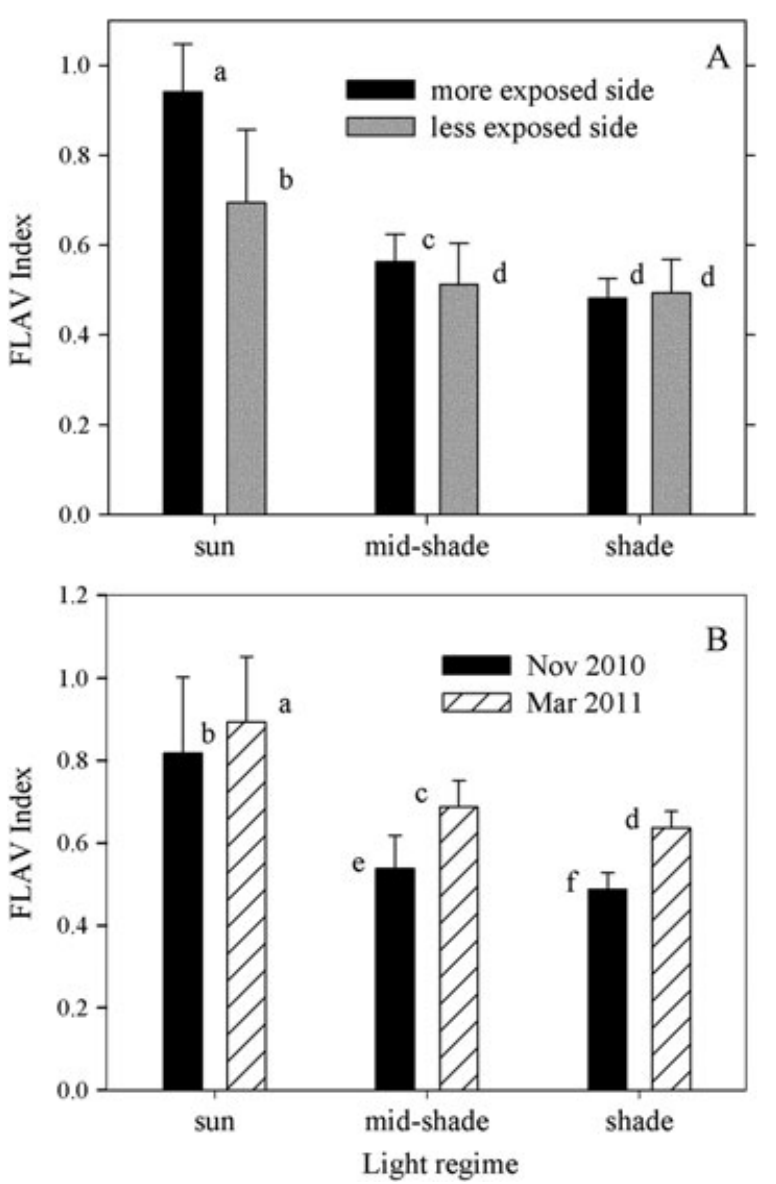

Figure 4. Non-destructive index of exocarp flavonols, FLAV index, determined by the Multiplex sensor on kiwifruits grown under different light regimes (sun, mid-shade, shade). (A) Mean ( \pm SD) values $(n=25)$ measured at harvest of the more exposed and less exposed fruit sides. (B) FLAV values $( \pm$ SD) averaged over both sides $(n=50)$ per light regime, measured at harvest (November 2010) and after 3-month storage at $0^{\circ} \mathrm{C}$ (March 2011). Bars accompanied by different letters are significantly different at $p<0.05$, based on a t-test analysis.

(RMSE) of $0.023 \mathrm{mg} / \mathrm{g}$ FW. Validation tests must be performed in the future on a large and independent dataset.

Comparing the flavonol fraction with the total polyphenol content determined by HPLC analysis in the kiwifruit exocarp extracts, it was found that they were fairly linearly related $\left(R^{2}=0.83\right)$ (data not shown). Therefore, the FLAV index also could be considered an indirect proxy of the total polyphenol concentrations.

\section{Non-destructive index of flavonols}

The mean values $( \pm S D$ ) of the FLAV index measured at harvest by the Mx sensor on the more-exposed and less-exposed sides of kiwifruits for each light regime are reported in Fig. 4A and Fig. $4 \mathrm{~B}$ shows FLAV averaged over both sides per light regime measured at harvest and after 3-month conservation at $0^{\circ} \mathrm{C}$.

We found markedly higher FLAV values in the fruits from the sun exposed positions compared with those from mid-shade and shade. Among the kiwifruits collected at the sunny sites, the two faces differed significantly in the FLAV index, due again to a different light exposition. The FLAV value of the less 
exposed sides of mid-shade was lower than that from the more exposed side and equal to both those under shade.

Storage at $0^{\circ} \mathrm{C}$ induced an increase in FLAV for all the light conditions. The increase was larger for shade and mid-shade than for full-sun-exposed kiwifruits, however, the differences observed at harvest in favour of sun fruits were maintained. The differences in the flavonol content as a function of the light regime estimated by the non-destructive method were in accordance with the results of the destructive analysis (Table 2).

The flavonol accumulation in fruit skins is dependent on the exposure to sunlight (Awad et al., 2001; Kolb et al., 2003), therefore, the FLAV index could be used as quantitative proxy of the exposure of kiwifruits to sun. This information is important because quality attributes, such as soluble solids, flesh firmness and chlorophyll, which are essential for long-term storage of kiwifruits, were found to be positively influenced by light exposure (Tombesi et al., 1993).

Flavonols protect internal tissues of plant organs by partially screening the UV solar radiation, but mainly by functioning as anti-oxidant against light-induced oxidative stress (Agati and Tattini, 2010). The main flavonoids found into the kiwifruit exocarp were rutin and quercetin $3-O-\beta-D$-rhamnoside (see Table 1), dihydroxy B-ring-substituted flavonoids, both possessing the structural requirements for effective detoxification of reactive oxygen species (Agati and Tattini, 2010). Flavonoids also play a significant role in plant resistance against insects and microbial infections (Treutter, 2005) and are important compounds in postharvest resistance of fruits and vegetables to pathogens (Lattanzio et al., 2006). Although little experimental evidence exists on the protective role of phenolics against fungal diseases in kiwifruits (Wurms, 2005), we can hypothesise that the larger the flavonol content of fruit skin the higher the integrity of fruit tissues. Interestingly, kiwifruits grown under denser canopies were more susceptible to develop Botrytis fungus (Michailides and Elmer, 2000), probably due to the reduced content in phenolic compounds determined by shade conditions (Greaves et al., 2001).

The content of flavonol in kiwifruits can, therefore, be considered an added value for the evaluation of the fruit quality and used as an index of suitability for preservation.

The observation regarding the increase in FLAV with $0^{\circ} \mathrm{C}$ storage is consistent with the rise in total phenolic compounds previously observed in kiwifruits after cold storage for 6 months (Tavarini et al., 2008). An increased synthesis of anthocyanins during low-temperature storage was also found in small fruits (Kalt et al., 1999) and oranges (Lo Piero et al., 2005). The increase in flavonols observed in kiwifruits can be explained as due to the overexpression induced by cold exposure of all the key enzyme genes involved in the phenylpropanoid biosynthetic pathway (Lo Piero et al., 2005).

\section{Applications}

This study showed that the kiwifruit could be a significant source of functional compounds for fruit-based ingredients containing polyphenols. The wastes from kiwifruits (skin and pomace) can account for up to $30 \%$ of the total kiwifruit crop (Schieber et al., 2001), normally discarded because of their unpleasant sensory attributes, such as intrinsic bitterness. On the other hand, as the anti-oxidant effects of kiwifruits in human body may prevent the development and deterioration induced by oxidative stress, much interest is turned to phenol food constituents and their capabilities to prevent or limit oxidative stress damage. In this context, the use of a non-destructive tool, such the $\mathrm{Mx}$ sensor, to select top-quality fruits can considerably improve the production of novel and functional foods. Fluorescence-based sensors can be used directly in the field on kiwifruits attached to the plant with the advantage of taking into account seasonal influences on fruit polyphenols, due to the variability of climatic conditions such as rainfall, temperature and irradiance. The Mx sensor could be easily integrated in online sorting devices using the index of polyphenol content as an additional quality parameter for storability.

\section{Acknowledgements}

The authors are grateful to the Azienda 'Camillo Pacini e Figli' (Rigoli, Pisa, Italy), for supplying the kiwifruit samples. The technical support of Andrea De Gregorio, Azienda Agricola II Quadrifoglio, Cisterna di Latina (Latina, Italy) is kindly acknowledged.

\section{References}

Agati G, Meyer S, Matteini P, Cerovic ZG. 2007. Assessment of anthocyanins in grape (Vitis vinifera L.) berries using a non-invasive chlorophyll fluorescence method. J Agric Food Chem 55: 1053-1061.

Agati G, Tattini M. 2010. Multiple functional roles of flavonoids in photoprotection. New Phytol 186: 786-793.

Awad MA, Wagenmakers PS, de Jager A. 2001. Effects of light on flavonoid and chlorogenic acid levels in the skin of 'Jonagold' apples. Sci Hortic-Amsterdam 88: 289-298.

Ben Ghozlen N, Cerovic ZG, Germain C, Toutain S, Latouche G. 2010. Nondestructive optical monitoring of grape maturation by proximal sensing. Sensors 10: 10040-10068.

Betemps DL, Fachinello JC, Galarca SP, Portela NM, Remorini D, Massai R, Agati G. 2012. Non-destructive evaluation of ripening and quality traits in apples using a multiparametric fluorescence sensor. J Sci Food Agr 92: 1855-1864.

Bilger W, Veit M, Schreiber L, Schreiber U. 1997. Measurement of leaf epidermal transmittance of UV radiation by chlorophyll fluorescence. Physiol Plant 101: 754-763.

Dawes HM, Keene JB. 1999. Phenolic composition of kiwifruit juice. J Agric Food Chem 47: 2398-2403.

Du GR, Li MJ, Ma FW, Liang D. 2009. Antioxidant capacity and the relationship with polyphenol and Vitamin C in Actinidia fruits. Food Chem 113: 557-562.

Ferguson AR. 1999. Kiwifruit cultivars: breeding and selection. Acta Hort 498: 43-52.

Fiorentino A, D'Abrosca B, Pacifico S, Mastellone C, Scognamiglio M, Monaco P. 2009. Identification and assessment of antioxidant capacity of phytochemicals from kiwi fruits. J Agric Food Chem 57: 4148-4155.

Greaves TJ, McGhie TK, Piller GJ, Meekings JS, Reglinski T, Klages K, Boldingh HL. 2001. Carbon status during leaf development in kiwifruit and subsequent resistance of wounded tissue to Botrytis cinerea. Crop Prot 20: 553-560.

Hagen SF, Solhaug KA, Bengtsson GB, Borge GIA, Bilger W. 2006. Chlorophyll fluorescence as a tool for non-destructive estimation of anthocyanins and total flavonoids in apples. Postharvest Biol Technol 41: 156-163.

Iwasawa H, Morita E, Yui S, Yamazaki M. 2011. Anti-oxidant effects of kiwi fruit in vitro and in vivo. Biol Pharm Bull 34: 128-134.

Ju ZG, Yuan YB, Liou CL, Xin SH. 1995. Relationships among phenylalanine ammonia-lyase activity, simple phenol concentrations and anthocyanin accumulation in apple. Sci Hortic 61: 215-226.

Kalt W, Forney CF, Martin A, Prior RL. 1999. Antioxidant capacity, vitamin $C$, phenolics, and anthocyanins after fresh storage of small fruits. $J$ Agric Food Chem 47: 4638-4644.

Kempler C, Kabaluk JT, Toivonen PMA. 1992. Effect of environment and harvest date on maturation and ripening of kiwifruit in British Columbia. Can J Plant Sci 72: 863-869.

Kolb C, Käser MA, Kopecky J, Zotz G, Reiderer M, Pfündel EE. 2001. Effects of natural intensities of visible and ultraviolet radiation on epidermal ultraviolet screening and photosynthesis in grape leaves. Plant Physiol 127: 863-875. 
Kolb CA, Kopecky J, Riederer M, Pfündel EE. 2003. UV screening by phenolics in berries of grapevine (Vitis vinifera). Func Plant Biol 30: 1177-1186.

Lattanzio V, Lattanzio VM, Cardinali A. 2006. Role of phenolics in the resistance mechanisms of plants against fungal pathogens and insects. Phytochem Adv Res 661: 23-67.

Lo Piero AR, Puglisi I, Rapisarda P, Petrone G. 2005. Anthocyanins accumulation and related gene expression in red orange fruit induced by low temperature storage. J Agric Food Chem 53: 9083-9088.

McGlone VA, Jordan RB, Seelye R, Martinsen PJ. 2002. Comparing density and NIR methods for measurement of kiwifruit dry matter and soluble solids content. Postharvest Biol Technol 26: 191-198.

Mendes Novo J, Iriel A, Lagorio MG. 2012. Modelling chlorophyll fluorescence of kiwi fruit (Actinidia deliciosa). Photochem Photobiol Sci 11: 724-730.

Merzlyak MN, Solovchenko AE, Gitelson AA. 2003. Reflectance spectral features and non-destructive estimation of chlorophyll, carotenoid and anthocyanin content in apple fruit. Postharvest Biol Technol 27: 197-211.

Michailides TJ, Elmer PAG. 2000. Botrytis gray mold of kiwifruit caused by Botrytis cinerea in the United States and New Zealand. Plant Dis $\mathbf{8 4}$ 208-223.

Montanaro G, Treutter D, Xiloyannis C. 2007. Phenolic compounds in young developing kiwifruit in relation to light exposure: Implications for fruit calcium accumulation. J Plant Interact 2: 63-69.
Remorini D, Tardelli F, Massai R, Guidi L, Degl'Innocenti E, Agati G. 2011. A non-destructive fluorescence method applied to the assessment of the quality of kiwifruit. Acta Hort 913: 547-552.

Schieber A, Stintzing FC, Carle R. 2001. By-products of plant food processing as a source of functional compounds - recent developments. Trends Food Sci Tech 12: 401-413.

Snelgar WP, Hopkirk G, Seelye RJ, Martin PJ, Manson PJ. 1998. Relationship between canopy density and fruit quality of kiwifruit. N Z J Crop Hortic Sci 26: 223-232.

Sun-Waterhouse D, Wen I, Wibisono R, Melton LD, Wadhwa S. 2009. Evaluation of the extraction efficiency for polyphenol extracts from by-products of green kiwifruit juicing. Int J Food Sci Tech 44: 2644-2652.

Tavarini S, Degl'Innocenti E, Remorini D, Massai R, Guidi L. 2008. Antioxidant capacity, ascorbic acid, total phenols and carotenoids changes during harvest and after storage of Hayward kiwifruit. Food Chem 107: 282-288.

Tombesi A, Antognozzi E, Palliotti A. 1993. Influence of light exposure on characteristics and storage life of kiwifruit. N Z J Crop Hort 21: 85-90.

Treutter D. 2005. Significance of flavonoids in plant resistance and enhancement of their biosynthesis. Plant Biol 7: 581-591.

Wurms KV. 2005. Susceptibility to Botrytis cinerea, and curing-induced responses of lytic enzymes and phenolics in fruit of two kiwifruit (Actinidia) cultivars. N Z J Crop Hort 33: 25-34. 